\title{
Treatment Strategies for Leber Hereditary Optic Neuropathy
}

Authors: Neringa Jurkute ${ }^{1}$, Joshua Harvey ${ }^{1,2}$, Patrick Yu-Wai-Man ${ }^{1,3,4,5}$

1. NIHR Biomedical Research Centre at Moorfields Eye Hospital and UCL Institute of Ophthalmology, London, United Kingdom

2. Ophthalmology Department, King's College Hospital, King's College Hospital NHS Trust, United Kingdom

3. Cambridge Eye Unit, Addenbrooke's Hospital, Cambridge University Hospitals, Cambridge, United Kingdom

4. MRC Mitochondrial Biology Unit, University of Cambridge, Cambridge, United Kingdom

5. Cambridge Centre for Brain Repair, Department of Clinical Neurosciences, University of Cambridge, Cambridge, United Kingdom

Corresponding author:

Professor Patrick Yu-Wai-Man. Institution, Address, Telephone, Fax and Email:

Cambridge Centre for Brain Repair, Department of Clinical Neurosciences, Forvie

Site, Robinson Way, University of Cambridge, Cambridge, CB2 OPY, United Kingdom.

E-mail:py237@cam.ac.uk

Tel: +441223331160

Fax: +44 1223331174 


\section{Purpose of review}

Leber hereditary optic neuropathy (LHON) is the most common primary mitochondrial DNA (mtDNA) disorder in the population and it carries a poor visual prognosis. In this article, we review the development of treatment strategies for LHON, the evidence base and the areas of unmet clinical need.

\section{Recent findings}

There is accumulating evidence that increasing mitochondrial biogenesis could be an effective strategy for protecting retinal ganglion cells (RGCs) in LHON. A number of clinical trials are currently investigating the efficacy of viral-based gene therapy for patients harbouring the m.11778G>A mtDNA mutation. For female LHON carriers of childbearing age, mitochondrial replacement therapy is being offered to prevent the maternal transmission of pathogenic mtDNA mutations.

\section{Summary}

Although disease-modifying treatment options remain limited, a better understanding of the underlying disease mechanisms in LHON is paving the way for complementary neuroprotective and gene therapeutic strategies for this mitochondrial optic nerve disorder.

Keywords: Gene therapy; idebenone; Leber hereditary optic neuropathy; mitochondrial disease; retinal ganglion cells. 


\section{Key Points}

- Leber hereditary optic neuropathy (LHON) is characterized by the preferential loss of retinal ganglion cells (RGCs) within the papillomacular bundle resulting in a dense central scotoma.

- Agents that increase mitochondrial biogenesis have shown promise in mitigating the deleterious effects of the LHON mitochondrial DNA (mtDNA) mutations in the cell models studied.

- Gene therapy based on allotopic expression of wild-type MTND4 is currently being investigated for patients carrying the m.11778G>A mtDNA mutation.

- Mitochondrial replacement therapy has been developed to prevent the maternal transmission of pathogenic mtDNA mutations. 


\section{Introduction}

LHON (OMIM 535000) is the most common primary mitochondrial DNA (mtDNA) disorder with a prevalence of 1 in 31000 - 50000 in Northern European populations and an estimated incidence of 1 in 1,000,000 in the Japanese population [1-3]. Three point mutations within the mitochondrial genome, m.3460G>A (MTND1) m.11778G>A (MTND4), and m.14484T>C (MTND6), account for about $90 \%$ of all LHON cases. These mtDNA mutations disrupt critical complex I subunits of the mitochondrial respiratory chain, causing impaired cellular ATP synthesis and increased production of reactive oxygen species (ROS) $[2,4]$. The remaining $10 \%$ of LHON cases harbour rarer pathogenic mtDNA mutations, which individually have been reported in only a limited number of patients worldwide [4-6]. Furthermore, there is a subset of patients with a LHON-like presentation who are not found to harbour any clear pathogenic variants on whole mtDNA sequencing. Some of these cases could be caused by a combination of multiple mtDNA variants that synergistically trigger retinal ganglion cell (RGC) loss or deleterious variants in nuclear-encoded genes, such as NDUFS2 [6-8--'].

Although the peak age of onset is between 15 and 35 years, patients with LHON have been reported from 2 to 87 years of age [5,9]. LHON typically presents with unilateral, painless, subacute, central visual loss with the fellow eye becoming involved within the following 6 months [10]. In about $25 \%$ of cases, there is simultaneous involvement of both optic nerves at first presentation [2,11]. An international consensus statement has defined 3 clinical stages in LHON based on the time from onset of visual loss, namely subacute (<6months), dynamic $(6-12$ months) and chronic (> 12 months) [12-"]. In the subacute phase, there is 
pseudopapilloedema due to swelling of the peripapillary retinal nerve fibre layer (RNFL), circumpapillary telangiectatic microangiopathy and vascular tortuosity. However, there is considerable heterogeneity in the appearance of the fundus and in about $20 \%$ of patients, the optic discs look normal, which can result in diagnostic delays [5]. As the disease progresses, there is rapid RGC axonal loss and pallor of the optic nerve head usually becomes evident within 6 weeks.

\section{Mitochondrial Neuroprotection - Idebenone}

Idebenone is a synthetic, short-chain analogue of ubiquinone, which is responsible for shuttling electrons from complexes I and II directly to complex III [13]. Idebenone has greater bioavailability compared with coenzyme Q10 (CoQ10) as it possesses a less lipophilic tail that allows it to penetrate the blood-brain barrier and mitochondrial membrane more readily [14]. The efficacy of idebenone was assessed in a randomized, double-blinded, placebo controlled study (RHODOS), which recruited 82 patients with LHON harboring one of the three common mtDNA mutations. Patients were randomised in a 2:1 ratio in favour of idebenone and a dose of $300 \mathrm{mg}$ three times a day was used over a treatment period of 24 weeks $[15,16]$. In addition to $\mathrm{RHODOS}$, the visual benefit of idebenone was assessed as part of a retrospective review of 103 patients treated with idebenone at varying doses and treatment duration [17]. Both these studies support a visual benefit in a proportion of patients treated with idebenone and the likelihood of a positive response was increased with earlier initiation of treatment. This is in keeping with the hypothesis that in the acute stage of LHON, a proportion of RGCs are functionally suppressed, but have not yet committed irreversibly cell death, and this pool of RGCs can be rescued if treatment is provided within a critical time window. Idebenone has been approved by the 
European Medicine Agency (EMA) to treat LHON and post-marketing studies are currently underway to collect additional safety and efficacy data [13].

\section{Other Neuroprotective Agents}

Two neuroprotective drugs, EPI-743 and MTP-131 (elamipretide), have been used for patients with mitochondrial disease $[18,19]$. In an open-labelled study, preliminary data of efficacy for EPI-743 was demonstrated in 5 patients with subacute LHON [20]. The safety and efficacy of a topical formulation of MTP-131 is currently being investigated in 12 patients with LHON with disease duration between 1 and 10 years (https://clinicaltrials.gov/ct2/show/NCT02693119, accessed $1^{\text {st }}$ of November 2018).

Cyclosporine A blocks apoptosis by inhibiting mitochondrial permeability transition and its therapeutic potential in LHON has been evaluated in 5 patients with subacute, unilateral LHON, who had been diagnosed within 6 months of disease onset [21,22-]. Cyclosporine A was not able to prevent second eye involvement at the treatment dose used $(2.5 \mathrm{mg} / \mathrm{kg} / \mathrm{day})$ [22-]. There is mounting evidence that mitochondrial dysfunction plays a key role in RGC loss in glaucoma [23,24--]. Interestingly, supplementation with nicotinamide adenine dinucleotide (NAD) precursors, which are derivatives of vitamin B3, was able to prevent the development of glaucoma in aged mice by improving mitochondrial metabolism and blocking proapoptotic pathways [23,24--]. Vitamin B3 and nicotinamide are therefore attractive neuroprotective molecules that could be pharmacologically modified to help promote neuronal survival in other optic neuropathies, including LHON.

\section{Improving Mitochondrial Biogenesis}

LHON is a complex disease and the phenotypic manifestation of the primary mtDNA mutation is influenced by secondary genetic factors, environmental triggers and hormonal influences $[25,26]$. The mitochondrial genome is a high copy number 
genome and the factors that control a cell's overall copy number are thought to be relevant to both physiological and pathological states [27]. Interestingly, unaffected LHON mutation carriers and normal healthy controls have a higher mtDNA copy number compared with affected patients with LHON [25]. By extrapolation, agents that improve mitochondrial biogenesis could therefore prove beneficial by providing a compensatory cellular response in the context of impaired mitochondrial oxidative phosphorylation. LHON fibroblasts treated with oestrogen derivatives show increased mtDNA copy and importantly, there is reduced ROS levels and increased cell survival [28]. These observations could also explain the marked sex bias in LHON with the preponderance for male carriers losing vision being due, at least partly, to lower circulating levels of oestrogens [28-30--]. Smoking is a major risk factor for visual loss in LHON and in keeping with this detrimental effect, LHON fibroblasts cultured in the presence of cigarette smoke condensates exhibit significantly decreased mtDNA copy number [31,32]. However, further experimental work is required to refine and test specific therapeutic agents that target mitochondrial biogenesis before moving into early phase clinical trials [33].

\section{Near-Infrared Light Therapy}

Photobiomodulation with near-infrared light-emitting diode (NIR-LED) arrays increases the production of cytochrome $c$ oxidase in cultured primary neurons and it reverses the reduction in enzymatic activity caused by metabolic inhibitors [34]. Recent experimental data in axotomy models of neurodegeneration look promising, but the translational application of this therapeutic paradigm to optic neuropathies poses a number of technical and practical challenges that will need to be overcome[35]. A previous study failed to recruit a sufficient number of patients to explore whether near-infrared light therapy could influence the visual prognosis in 
LHON (https://clinicaltrials.gov/ct2/show/NCT01389817, accessed $1^{\text {st }}$ of November 2018).

\section{Mitophagy Modulation}

Mitophagy refers to the selective autophagocytosis of mitochondria and this tightly regulated process allows the elimination of damaged mitochondria that would otherwise compromise the cell's survival. The balance between mitochondrial biogenesis and degradation has been investigated in LHON cybrid models and the available data indicates that mitophagy is dysregulated with impaired mitochondrial function and cell survival [36,37']. Based on these findings, activation of mitophagy by pharmacological means is being explored as a potential therapeutic strategy for mitochondrial diseases.

\section{Gene Therapy}

The eye represents an ideal target organ for gene therapy given the relative ease of access for direct delivery of viral vectors [38]. A major challenge in the treatment of mitochondrial disease is the double-membrane nature of the mitochondrial compartment, which complicates the efficient import of exogenous proteins. To bypass this physical barrier, an alternative strategy is to express the replacement wild-type gene in the nuclear compartment and the resulting protein is designed to contain a mitochondrial targeting sequence, which directs for its importation into mitochondria [39]. Following the successful demonstration that allotopic expression of wild-type MTND4 could rescue the phenotype both in cell and animal models of LHON, human clinical trials have been initiated using modified adeno-associated virus (AAV) vectors to deliver the gene construct [40-43--]. These groundbreaking studies have shown the safety of this approach and the preliminary results obtained 
are encouraging indicating the preservation of RGCs and a functional visual benefit in a proportion of treated eyes.

\section{Mitochondrial Replacement Therapy}

As the mitochondrial genome shows strict maternal inheritance, preventing the transmission of pathogenic mtDNA mutations from mother to child is a major area of research for a number of research groups worldwide. Two main in vitro fertilization (IVF) techniques have been developed, namely, pronuclear transfer and maternal spindle transfer, both of which require the contribution of a donor egg that carries only wild-type mtDNA [44]. The term "three-parent embryo" is frequently used in the lay press as the mitochondrial genome of the resulting embryo is derived from the donor egg whereas the nuclear genome is inherited from the biological parents. In 2015, mitochondrial replacement therapy was approved by both Houses of Parliament in the United Kingdom as a justifiable reproductive option with the caveat that it should only be carried out in a recognised centre of excellence and with longterm follow-up of the children born using this approach [45]. In contrast, the US Food and Drug Administration (FDA) has concluded that more experimental and safety data are needed before advocating mitochondrial replacement therapy as reproductive option for mitochondrial disease $[33,45]$. As the legal and ethical implications of mitochondrial donation was being openly debated, the media reported that maternal spindle transfer had resulted in the birth of a healthy baby boy who carried low levels of the m.8993T>G mtDNA mutation in MTAP6 that had resulted in the premature death of two siblings from Leigh syndrome [46"]. Mitochondrial replacement therapy remains controversial and both the regulatory framework and the consequences of modifying the germline need to be carefully considered [45].

\section{Regenerative Medicine}


The seminal discovery that somatic cells could be reprogrammed into pluripotent stem cells, so called induced pluripotent stem cells (iPSCs), has transformed the scope for regenerative medicine, in particular for progressive neurodegenerative diseases [47]. Although the generation of iPSCs carrying pathogenic mtDNA LHON mutations is now technically straightforward, their differentiation into RGCs and the generation of a homogenous population in sufficient quantity for experimental needs still remain challenging [48-]. Other hurdles that will need to be overcome before considering the use of iPSC-derived RGCs in LHON and other optic neuropathies are the methods required to deliver the cells in a viable state to the right retinal location and providing the guiding signals needed to allow the axonal projections of the transplanted cells to make the correct retinotopic connections [49-51]. A summary of the clinical studies and treatment strategies for LHON is listed in Table 1.

\section{Conclusions}

LHON is a rapidly progressive, blinding disorder that typically affects young adults in their prime. The management of LHON remains largely supportive, but rapid advances in drug discovery, gene therapy and stem cell technology are providing researchers with new tools for rescuing RGCs in this disorder with the aim of stabilising vision and improving the visual outcome. Central to these endeavours is the need to ensure patient safety both in early phase experimental trials and as part of long-term follow-up studies. 


\section{Acknowledgements}

None.

\section{Financial support and sponsorship}

PYWM is supported by a Clinician Scientist Fellowship Award (G1002570) from the Medical Research Council (UK), and also receives funding from Fight for Sight (UK), the Isaac Newton Trust (UK), the UK National Institute of Health Research (NIHR) as part of the Rare Diseases Translational Research Collaboration, and the NIHR Biomedical Research Centre based at Moorfields Eye Hospital NHS Foundation Trust and UCL Institute of Ophthalmology. The views expressed are those of the author(s) and not necessarily those of the NHS, the NIHR or the Department of Health.

\section{Conflicts of interest}

PYWM holds a consultancy agreement with GenSight Biologics (Paris, France). NJ and $\mathrm{JH}$ none. 


\section{References}

1. Yu-Wai-Man P, Griffiths PG, Brown DT et al. The epidemiology of Leber hereditary optic neuropathy in the North East of England. Am J Hum Genet 2003, 72:333-9.

2. Meyerson C, Van Stavern G, McClelland C. Leber hereditary optic neuropathy: current perspectives. Clin Ophthalmol 2015, 9:1165-1176.

3. Ueda K, Morizane Y, Shiraga F et al. Nationwide epidemiological survey of Leber hereditary optic neuropathy in Japan. J Epidemiol 2017, 27:447-450.

4. Yu-Wai-Man P, Griffiths PG, Chinnery PF. Mitochondrial optic neuropathies - Disease mechanisms and therapeutic strategies. Prog Retin Eye Res 2011, 30:81-114.

5. Yu-Wai-Man P, Votruba M, Moore AT et al. Treatment strategies for inherited optic neuropathies: past, present and future. Eye 2014, 28:521-537.

6. Jurkute $\mathrm{N}$, Majander A, Bowman R et al. Clinical utility gene card for: inherited optic neuropathies including next-generation sequencing-based approaches. Eur J Hum Genet 2018, doi:10.1038/s41431-018-0235-y.

7. Caporali L, lommarini L, La Morgia C et al. Peculiar combinations of individually nonpathogenic missense mitochondrial DNA variants cause low penetrance Leber's hereditary optic neuropathy. PLOS Genet 2018, 14:e1007210.

- This paper provides evidence that in the absence of a clearly established primary mtDNA mutation, specific combinations of missense mtDNA variants could be sufficiently deleterious to impair mitochondrial function causing LHON.

8. Gerber S, Ding MG, Gérard X et al. Compound heterozygosity for severe and hypomorphic NDUFS2 mutations cause non-syndromic LHON-like optic neuropathy. J Med Genet 2017, $54: 346-356$.

9. Majander A, Bowman R, Poulton J et al. Childhood-onset Leber hereditary optic neuropathy. Br J Ophthalmol 2017, 101:bjophthalmol-2016-310072. 
10. Hwang TJ, Karanjia R, Moraes-Filho MN et al. Natural History of Conversion of Leber's Hereditary Optic Neuropathy: A Prospective Case Series. Ophthalmology 2017, 124:843-850.

- In this interesting prospective study, the authors describe the preclinical changes that were observed in a group of LHON carriers prior to disease conversion.

11. Borrelli E, Triolo G, Cascavilla ML et al. Changes in Choroidal Thickness follow the RNFL Changes in Leber's Hereditary Optic Neuropathy. Nature 2016, doi:10.1038/srep37332.

12. Carelli V, Carbonelli M, de Coo IF et al. International Consensus Statement on the Clinical and Therapeutic Management of Leber Hereditary Optic Neuropathy. J Neuro-Ophthalmology 2017, 37:371-381.

m. This consensus statement provides guidance on the management of LHON, in particular the indications for using idebenone in this disorder.

13. Jurkute N, Yu-Wai-Man P. Leber hereditary optic neuropathy: bridging the translational gap. 2017, doi:10.1097/ICU.0000000000000410.

14. Hargreaves IP. Coenzyme Q10 as a therapy for mitochondrial disease. Int J Biochem Cell Biol 2014, 49:105-111.

15. Klopstock T, Yu-Wai-Man P, Dimitriadis $\mathrm{K}$ et al. A randomized placebo-controlled trial of idebenone in Leber's hereditary optic neuropathy. Brain 2011, doi:10.1093/brain/awr170.

16. Klopstock T, Metz G, Yu-Wai-Man P et al. Persistence of the treatment effect of idebenone in Leber's hereditary optic neuropathy. Brain 2013, 136:e230.

17. Carelli V, Morgia C La, Valentino ML et al. Idebenone treatment in Leber's hereditary optic neuropathy. Brain 2011, 134:1-5.

18. Karaa A, Haas R, Goldstein A et al. Randomized dose-escalation trial of elamipretide in adults with primary mitochondrial myopathy. Neurology 2018, 90:e1212-e1221.

19. Sadun AA. Effect of EPI-743 on the Clinical Course of the Mitochondrial Disease Leber Hereditary Optic Neuropathy. Arch Neurol 2012, 69:331. 
20. Sadun AA, Chicani CF, Ross-Cisneros FN et al. Effect of EPI-743 on the Clinical Course of the Mitochondrial Disease Leber Hereditary Optic Neuropathy. Arch Neurol 2012, 69:331-338.

21. Waldmeier P, Zimmermann K, Qian T et al. Cyclophilin D as a Drug Target. Curr Med Chem 2003, 10:1485-1506.

22. Leruez S, Verny C, Bonneau D et al. Cyclosporine A does not prevent second-eye involvement in Leber's hereditary optic neuropathy. Orphanet J Rare Dis 2018, 13:33.

- This study highlights the difficulty in preventing fellow eye involvement in LHON once the disease process has started.

23. Williams PA, Harder JM, Foxworth NE et al. Glaucoma in Aged Mice. Science (80- ) 2017, $760: 756-760$.

24. Williams PA, Harder JM, Cardozo BH et al. Nicotinamide treatment robustly protects from inherited mouse glaucoma. Commun Integr Biol 2018, $11: \mathrm{e} 1356956$.

mese two studies, [23] and [24], demonstrate the neuroprotective role of the NAD precursor nicotinamide in a murine DBA/2J glaucoma model.

25. Giordano C, lommarini L, Giordano L et al. Efficient mitochondrial biogenesis drives incomplete penetrance in Leber's hereditary optic neuropathy. Brain 2014, 137:335-353.

26. Lambertini L, Byun HM. Mitochondrial Epigenetics and Environmental Exposure. Curr Env Heal Rep 2016, 3:214-224.

27. Hirano M. Weighing in on Leber hereditary optic neuropathy: effects of mitochondrial mass. Brain 2014, 137:308-9.

28. Giordano C, Montopoli M, Perli E et al. Oestrogens ameliorate mitochondrial dysfunction in Leber's hereditary optic neuropathy. Brain 2011, 134:220-234.

29. Bianco A, Bisceglia L, Russo L et al. High Mitochondrial DNA Copy Number Is a Protective Factor From Vision Loss in Heteroplasmic Leber's Hereditary Optic Neuropathy (LHON). Investig Opthalmology Vis Sci 2017, 58:2193. 
m The main observation from these two studies ([28] and [29]) is that a higher mtDNA copy number seems to protect LHON carriers from losing vision, implying that boosting mitochondrial biogenesis could be a therapeutic target in LHON.

30. Pisano A, Preziuso C, lommarini $L$ et al. Targeting estrogen receptor $\beta$ as preventive therapeutic strategy for Leber's hereditary optic neuropathy. Hum Mol Genet 2015, 24:ddv396.

31. Giordano L, Deceglie S, d'Adamo P et al. Cigarette toxicity triggers Leber's hereditary optic neuropathy by affecting mtDNA copy number, oxidative phosphorylation and ROS detoxification pathways. Cell Death Dis 2015, 6:e2021.

32. Wenz T, Williams SL, Bacman SR et al. Emerging therapeutic approaches to mitochondrial diseases. Dev Disabil Res Rev 2010, 16:219-29.

33. Kim US, Jurkute N, Yu-Wai-Man P. Leber Hereditary Optic Neuropathy — Light at the End of the Tunnel? Asia-Pacific J Ophthalmol 2018, 0:1-4.

34. Eells JT, Wong-Riley MTT, VerHoeve $\mathrm{J}$ et al. Mitochondrial signal transduction in accelerated wound and retinal healing by near-infrared light therapy. Mitochondrion 2004, 4:559-567.

35. Beirne K, Rozanowska M, Votruba M. Photostimulation of mitochondria as a treatment for retinal neurodegeneration. Mitochondrion 2017, 36:85-95.

36. Sharma LK, Tiwari M, Rai NK et al. Mitophagy activation repairs Leber's Hereditary Optic Neuropathy associated mitochondrial dysfunction and improves cell survival. Hum Mol Genet 2018, doi:10.1093/hmg/ddy354.

37. Dombi E, Diot A, Morten $\mathrm{K}$ et al. The m.13051G $>$ A mitochondrial DNA mutation results in variable neurology and activated mitophagy. Neurology 2016, 86:1921-3.

- The main observation from these two studies ([36] and [37]) is that mtDNA LHON mutations have an impact on mitochondrial turnover by impairing autophagy. Interestingly, pharmacological activiation of autophagy was found to improve cell survival in the LHON cybrid models used. 
38. Yu-Wai-Man P, Chinnery PF. Leber Hereditary Optic Neuropathy. University of Washington, Seattle; 1993.

39. Cwerman-Thibault H, Augustin S, Ellouze S et al. Leber Hereditary Optic Neuropathy as the first candidate for a clinical trial. C R Biol 2014, 337:193-206.

40. Wan X, Pei H, Zhao M et al. Efficacy and Safety of rAAV2-ND4 Treatment for Leber's Hereditary Optic Neuropathy. Sci Rep 2016, 6:1-10.

41. Guy J, Feuer WJ, Davis JL et al. Gene Therapy for Leber Hereditary Optic Neuropathy: Lowand Medium-Dose Visual Results. Ophthalmology 2017, 124:1621-1634.

42. Guy J, Feuer WJ, Davis JL et al. Gene Therapy for Leber Hereditary Optic Neuropathy. Ophthalmology 2017, 124:1621-1634.

43. Vignal C, Uretsky S, Fitoussi S et al. Safety of rAAV2/2-ND4 Gene Therapy for Leber Hereditary Optic Neuropathy. Ophthalmology 2018, 0.

- Clinical trials [40-43] are currently underway to assess whether allotopic expression of the wild-type MTND4 gene can rescue RGCs and improve the visual prognosis in patients with LHON carrying the m.11778G $>$ A mutation.

44. Hyslop LA, Blakeley P, Craven L et al. Towards clinical application of pronuclear transfer to prevent mitochondrial DNA disease. Nature 2016, 534:383-386.

45. Newson AJ, Wilkinson S, Wrigley A. Ethical and legal issues in mitochondrial transfer. EMBO Mol Med 2016, 8:589-91.

46. Zhang J, Liu H, Luo S et al. Live birth derived from oocyte spindle transfer to prevent mitochondrial disease. Reprod Biomed Online 2017, 34:361-368.

- This is the first report of a succesful live birth arising from mitochondrial replacement therapy using the spindle transfer technique.

47. Takahashi K, Yamanaka S. Induction of Pluripotent Stem Cells from Mouse Embryonic and Adult Fibroblast Cultures by Defined Factors. Cell 2006, 126:663-676. 
48. Wong RCB, Lim SY, Hung SSC et al. Mitochondrial replacement in an iPSC model of Leber's hereditary optic neuropathy. Aging (Albany NY) 2017, 9:1341-1350.

- The authors of this study made use of iPSC technology to generate RGCs carrying two homoplasmic mtDNA mutations, namely, m.4160T>C (MTND1) and m.14484T>C (MTND6). Cybrid technology was used to replace the mutant mtDNA population with wild-type mtDNA and this rescue strategy was succesful in reducing ROS levels and blocking apoptosis.

49. Tanaka T, Yokoi T, Tamalu F et al. Generation of Retinal Ganglion Cells With Functional Axons From Mouse Embryonic Stem Cells and Induced Pluripotent Stem Cells. Investig Opthalmology Vis Sci 2016, 57:3348.

50. Tanaka T, Yokoi T, Tamalu F et al. Generation of retinal ganglion cells with functional axons from human induced pluripotent stem cells. Sci Rep 2015, 5:8344.

51. Teotia P, Chopra DA, Dravid SM et al. Generation of Functional Human Retinal Ganglion Cells with Target Specificity from Pluripotent Stem Cells by Chemically Defined Recapitulation of Developmental Mechanism. Stem Cells 2017, 35:572-585. 